\title{
The transformation dynamics towards equilibrium in non-equilibrium w/w/o double emulsions
}

\author{
Youchuang Chao, ${ }^{1,2}$ Sze Yi Mak, ${ }^{1,2}$ and Ho Cheung Shum ${ }^{1,2, a)}$ \\ ${ }^{1}$ Department of Mechanical Engineering, The University of Hong Kong, Pokfulam Road, Hong Kong, China \\ ${ }^{2} \mathrm{HKU}$-Shenzhen Institute of Research and Innovation (HKU-SIRI), Shenzhen, Guangdong 518000, China
}

(Received 20 September 2016; accepted 20 October 2016; published online 31 October 2016)

\begin{abstract}
We use a glass-based microfluidic device to generate non-equilibrium water-in-water-in-oil (w/w/o) double emulsions and study how they transform into equilibrium configurations. The method relies on using three immiscible liquids, with two of them from the phase-separated aqueous two-phase systems. We find that the transformation is accompanied by an expansion rim, while the characteristic transformation speed of the rim mainly depends on the interfacial tension between the innermost and middle phases, as well as the viscosity of the innermost phase when the middle phase is nonviscous. Remarkably, the viscosity of the outermost phase has little effect on the transformation speed. Our results account for the dynamics of non-equilibrium double emulsions towards their equilibrium structure and suggest a possibility to utilize the non-equilibrium drops to synthesize functional particles. Published by AIP Publishing. [http://dx.doi.org/10.1063/1.4966902]
\end{abstract}

The controlled generation of double emulsions continues to be of interest for the scientific community due to the applications in various fields like food industry, cosmetics, and materials. ${ }^{1-4}$ Microfluidic methods provide an excellent platform to produce multiple emulsions because of the high degree of flexibility and control. ${ }^{5-7}$ Depending on the interfacial tensions between the three phases of the double emulsions, three morphologies can form: (i) the droplets are separated by the outermost phase (non-engulfing), (ii) one of the phases completely engulfs the other one (complete engulfing), or (iii) Janus droplets (partial engulfing). ${ }^{8,9}$ However, double emulsions produced by microfluidics or the scalable batch technology are not always in their equilibrium state, and the transition from the non-equilibrium state to the equilibrium state is very fast and, consequently, rarely captured. As a result, this fast transition prevents us from unraveling its detailed kinetics, or utilizing it to form non-equilibrium droplets. De-wetting strategies, induced by temperature changes, light, evaporation, and phase separation, have been applied to trigger this transition for fabricating complex emulsions. ${ }^{10-16}$ The geometries of double emulsions can be altered among the different configurations by varying the interfacial tensions using stimuli-responsive and cleavable surfactants. ${ }^{14}$ The regulated and complex Janus droplets can also be fabricated by co-solvent-evaporation-driven phase separation and dewetting. ${ }^{15}$ The spreading coefficients, $S_{i}=\gamma_{j k}-\left(\gamma_{i j}+\gamma_{i k}\right)$, are often adopted as criteria to predict the final architectures of the double emulsion drops. These coefficients determine the equilibrium state from the perspective of minimized interfacial energy of the three-phase system. $S_{i}<0$ indicates that the formation of an interface between the two phases $j$ and $k$ is energetically favored. ${ }^{8,9,17}$ The configuration of the innermost droplets in non-equilibrium double emulsions is also induced sometimes hydrodynamically and frozen by photo-curing the droplets into particles. ${ }^{18,19}$ An understanding of the kinetics

\footnotetext{
${ }^{\text {a) }}$ Author to whom correspondence should be addressed. Electronic mail: ashum@hku.hk
}

of the transition would enable the engineering of different particle morphologies; however, so far, most experimental efforts have focused on the relationship between the spreading coefficients and the equilibrium morphology of the double emulsion drops. The dynamics from the energetically unfavorable state to the equilibrium state, especially how the innermost droplet de-wets from the host droplet, still remain unclear. A physical understanding of the non-equilibrium double emulsion drops towards their equilibrium state allows the design of double emulsion drops with desired structure.

In this paper, we report a microfluidic approach to generate non-equilibrium water-in-water-oil (w/w/o) double emulsions and how they transform into the equilibrium state. We focus on the transformation dynamics from the unstable "complete engulfing" to a stable "complete engulfing" configuration. The innermost and middle phases are made up of the phase-separated aqueous two-phase systems (ATPS), ${ }^{20,21}$ and the outermost phase is silicone oil. Interestingly, the transformation is accompanied by an expanding rim, and the characteristic timescale of the transformation can be as slow as tens of seconds when the interfacial tension between the two aqueous phases is ultralow $(\sim 1 \mu \mathrm{N} / \mathrm{m})$. By performing a simple scaling analysis, we show that the transformation speed is mainly determined by the interfacial tension between the two aqueous phases and the viscosity of the innermost fluid when the middle phase is non-viscous. The viscosity of the outermost phase has little effect on the transformation speed. The understanding of the observed dynamics suggests a route to predict the lifetime of the non-equilibrium double emulsions and thus facilitates the use of the intermediate droplets as templates to fabricate particles with tunable morphologies.

The microfluidic device consists of a cylindrical glass capillary with an outer diameter of $1 \mathrm{~mm}$ and two square glass capillaries with inner side lengths of $1.75 \mathrm{~mm}$ and $1.05 \mathrm{~mm}$, respectively. The cylindrical capillary is coaxially aligned with the small square capillary tube, and then both are inserted into the large square capillary tube. The inner 
cylindrical capillary and the middle square capillary are tapered, by a micropipette puller (Sutter P-97), into tips with the approximate inner diameters of $50 \mu \mathrm{m}$ and $100 \mu \mathrm{m}$, respectively. The outer cylindrical capillary is treated with trimethoxy(octadecyl)saline to render its surface hydrophobic, preventing wetting of the aqueous phases on the capillary wall. We pump aqueous solutions of polyethylene glycol (PEG $M_{w}=8000,10$ wt. $\%-22$ wt. $\%$ PEG, or $M_{w}=4000,10$ wt. \%25 wt. \%) and salts $\left(\mathrm{C}_{6} \mathrm{H}_{5} \mathrm{Na}_{3} \mathrm{O}_{7} \cdot 2 \mathrm{H}_{2} \mathrm{O}, 10\right.$ wt. \%-22 wt. \%, or $\mathrm{Na}_{2} \mathrm{CO}_{3}, 5$ wt. \%-10 wt. \%) into the innermost and middle capillaries, respectively. The third liquid is silicone oil with a wide range of viscosity ( $5 \mathrm{cSt}-1000 \mathrm{cSt}$ ) and flows through the outermost capillary, as shown in Fig. 1(a).

The two aqueous solutions separated from the equilibrated aqueous two-phase systems [see Figure S1 in the supplementary material], whose interfacial tension can be easily tuned by changing the concentrations of the two phases, provide great flexibility in tuning the interfacial tensions between the innermost and middle phases. ${ }^{22,23} \mathrm{By}$ decreasing the concentrations of the two aqueous phases, we vary the interfacial tension from $2.68 \mathrm{mN} / \mathrm{m}$ to around $1 \mu \mathrm{N} /$ $\mathrm{m}$, which are obtained experimentally by a spinning drop tensiometer (KRÜSS, SITE100). The interfacial tension differences between either of the aqueous phases and the silicone oil are less than $4 \mathrm{mN} / \mathrm{m}$, as obtained by the pendant drop method. ${ }^{24,25}$ Besides, the viscosities of the PEG-rich phase and salt-rich phase vary from $11 \mathrm{mPa}$ s to $565 \mathrm{mPa} \mathrm{s}$, and $1.8 \mathrm{mPa}$ s to $6.1 \mathrm{mPa}$, respectively, as measured by a viscometer (RheoSense $\mu$ VISC). The physical properties of the two aqueous phases are measured at room temperature $(\mathrm{T}=298 \mathrm{~K})$ [see Table S1 in the supplementary material].

The three-phase drops directly formed inside the microfluidic device described is energetically unfavorable, due to the high interfacial tension, $\gamma_{S O}$, between the salt-rich aqueous solution and silicone oil relative to the sum of interfacial tensions between the PEG-rich aqueous solution and silicone
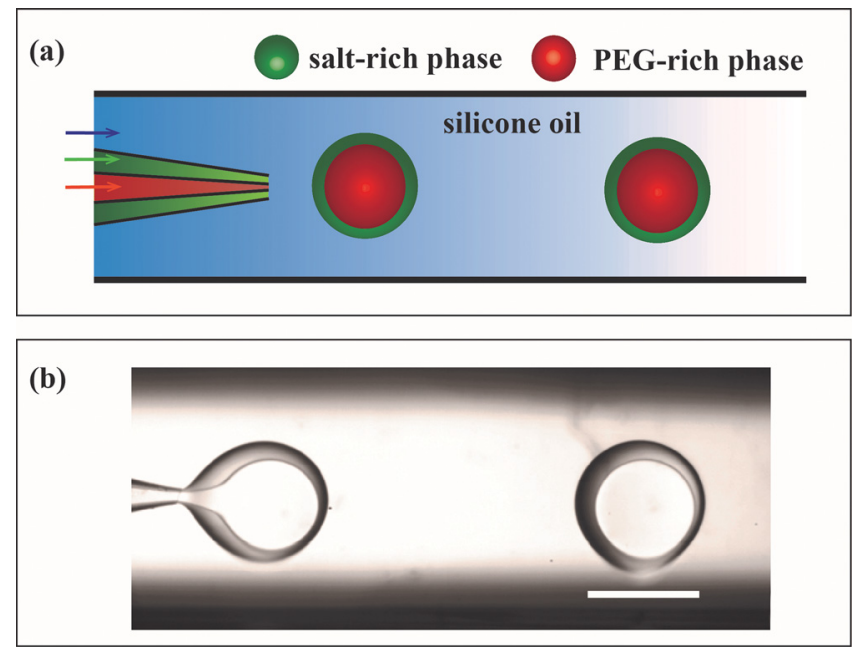

FIG. 1. (a) Schematic illustration of the glass-capillary microfluidic device used for the formation of non-equilibrium w/w/o double emulsion drops. (b) Optical microscope image showing the formation of non-equilibrium w/w/o double emulsion drops. The compound jet consisting of an inner aqueous core and middle aqueous annulus is induced to break up at the tip simultaneously. Flow rates of the inner PEG-rich phase, middle salt-rich phase, and outer oil phase are $0.8 \mathrm{ml} / \mathrm{h}, 0.8 \mathrm{ml} / \mathrm{h}$, and $15 \mathrm{ml} / \mathrm{h}$, respectively. The scale bar is $1 \mathrm{~mm}$. oil $\left(\gamma_{P O}\right)$, and between the salt-rich aqueous solution and PEGrich aqueous solution $\left(\gamma_{S P}\right): \gamma_{S O}>\gamma_{P O}+\gamma_{S P}$. However, due to the low interfacial tensions between the two aqueous phases, uniform w/w/o double emulsion drops cannot be generated spontaneously. ${ }^{26,27}$ To achieve uniform w/w/o drops, we first form a compound jet with one aqueous core jet surrounded by another aqueous annulus jet by increasing the flow rate of the inner aqueous phase. Afterwards, the compound jet is induced by the high interfacial tension between the middle aqueous phase and the outermost silicone oil to break up simultaneously to form w/w/o drops. ${ }^{28}$ After the non-equilibrium double emulsion drops are formed, we stop the pumps and allow their configurations to evolve with time. We monitor the dynamic behaviors of the non-equilibrium w/w/o drops using a high speed camera (Phantom V9.1, FASTCAM SA4, Photron) coupled with an inverted microscope (Motic AE2000). Using this co-flow configuration, we can produce uniform nonequilibrium w/w/o double emulsion drops, in which the core and shell radii are controlled to $\sim 0.7 \mathrm{~mm}$ and $\sim 1 \mathrm{~mm}$, respectively, as shown in Fig. 1(b).

The non-equilibrium double emulsion drops with a "complete engulfing" eventually transform into equilibrium configurations with another "complete engulfing" morphology. Interestingly, the transformation process is always accompanied by a fast expanding rim, indicating that the shell de-wets from the core droplet. The whole evolution process finishes in about $200 \mathrm{~ms}$ for the non-equilibrium double emulsion drops consisting of an inner drop of water with $16 \mathrm{wt}$. \% PEG $\left(M_{w}=4000\right)$, a middle shell of water with $5 \mathrm{wt}$. \% $\mathrm{Na}_{2} \mathrm{CO}_{3}$ and a continuous phase of silicone oil (1000 cSt). A variety of intermediate states with "partial engulfing" configuration (Janus drops) also exist during the transformation, as shown in Figs. 2(a) and 2(b). In addition, the resulting time sequence of the images also reveals that the transformation process is three-dimensional, with regions in and out of focus [see Movies S1, S2, and S3 in the supplementary material].

To quantify this transformation behavior, we measure the radius of the de-wetting rim and plot the result as a function of time. However, the recorded images are two-dimensional, so the measured chord length $\left(R_{\text {chord }}\right)$ needs to be converted into the radius of the rim $\left(R_{\text {rim }}\right)$, as shown in Fig. 3(a). The relationship between the rim radius $\left(R_{\text {rim }}\right)$ and the measured chord length $\left(R_{\text {chord }}\right)$ is $R_{\text {rim }}=\left(R_{\text {shell }}\right) \arcsin \left(R_{\text {chord }} / R_{\text {shell }}\right)$, where $R_{\text {shell }}$ is the radius of the shell droplet, as shown in Fig. 3(b). We observe that the radius of the rim expands almost linearly with time, and thus the speed $\left(V_{\text {rim }}\right)$ is constant and equals to $(1.66 \pm 0.08) \mathrm{mm} / \mathrm{s}$ for the non-equilibrium double emulsion drops [Fig. 3(c)].

However, various factors influence the transformation speed of the non-equilibrium w/w/o double emulsion drops. We first consider the viscous effects from the outermost oil phase and middle aqueous phase. Intuitively, the viscous outer fluid should impede the transformation; however, the experimental results show that the speed of the transformation process does not depend appreciably on the viscosity of the outer oil phase ( $5 \mathrm{cSt}-1000 \mathrm{cSt}$ in our experiment), as shown in Fig. 3(d). Furthermore, we assume that viscous stress imposed by the middle aqueous phase can be neglected due to the low viscosity of the middle phase of a salt solution, $\mu_{\text {salt }}$, from $1.8 \mathrm{mPa}$ s to $6 \mathrm{mPa} \mathrm{s}$, as compared with the high 

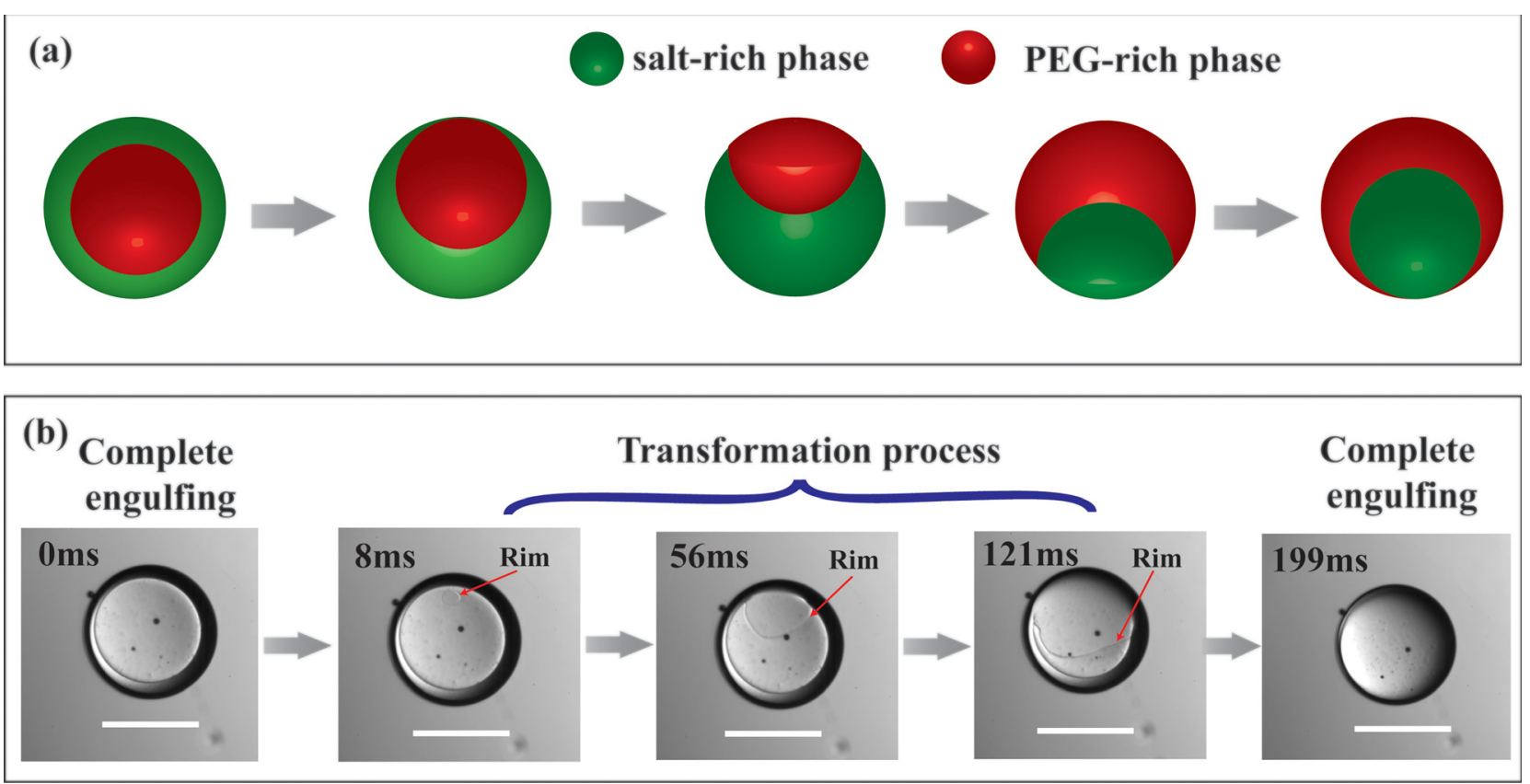

FIG. 2. Configuration transformation. (a) The schematic of the evolution of the non-equilibrium double emulsion drops with one "complete engulfing" morphology into another "complete engulfing" morphology. (b) Typical micrographs of the evolution of a non-equilibrium double emulsion drops consisting of an inner drop of water with 16 wt. \% PEG $\left(M_{w}=4000\right)$, a middle shell of water with 5 wt. $\% \mathrm{Na}_{2} \mathrm{CO}_{3}$, and a continuous phase of silicone oil (1000 cSt). Red arrows mark the de-wetting rims. See Figures S2 and S3 in the supplementary material for the side view of the transformation and similar transformation with paraffin liquid as the continuous phase. The scale bar is $1 \mathrm{~mm}$.

viscosity of innermost PEG-rich aqueous phase, $\mu_{\mathrm{PEG}}$, from $11 \mathrm{mPa}$ s to $565 \mathrm{mPa}$ s [see Table $\mathrm{S} 1$ in the supplementary material].

Based on these observations, we suggest that the transformation speed of the non-equilibrium w/w/o double emulsion drops is mainly affected by the interfacial tension and the viscous stress from the innermost phase. The buoyancy effect is negligible for the transformation, as indicated by the small local Bond number $\left(B o=\Delta \rho g l^{2} / \gamma<1\right)$, where $\Delta \rho$ is the density difference, $g$ is the gravitational constant, (a)

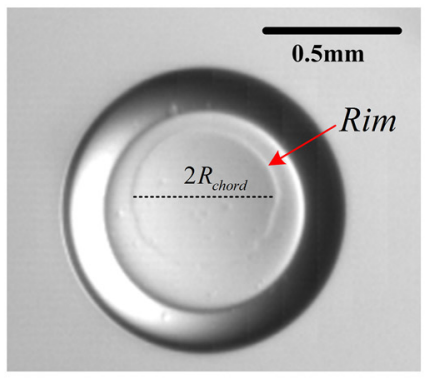

(c)

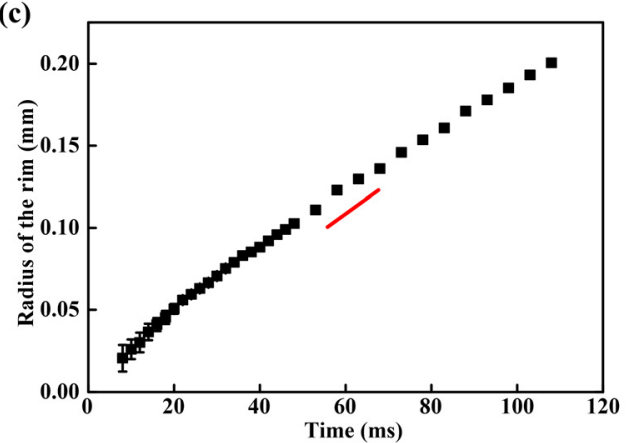

(b)

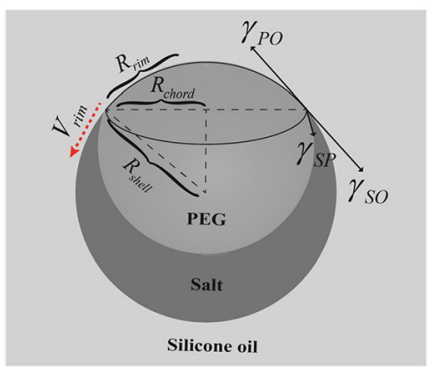

(d)

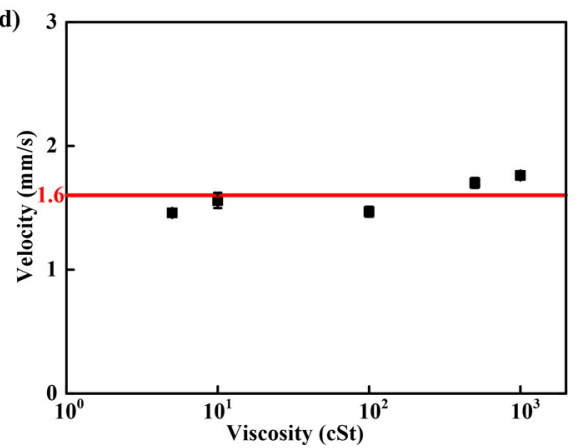

FIG. 3. (a) Typical optical micrograph of an expanding rim (top view). (b) Schematic of the intermediate non-equilibrium double emulsion drops (side view) and interfacial tensions $\left(\gamma_{S O}, \gamma_{P O}\right.$, and $\left.\gamma_{S P}\right)$ of the three different interfaces. The rim radius $\left(R_{\text {rim }}\right)$ can be calculated from the measured chord length $\left(R_{\text {chord }}\right)$ using this expression: $R_{\text {rim }}=\left(R_{\text {shell }}\right) \arcsin \left(R_{\text {chord }} / R_{\text {shell }}\right)$, where $R_{\text {shell }}$ denotes the radius of the shell droplet. Due to the optical resolution, the thickness of the rim is not considered. The dashed arrow (red) indicates the expanding direction of rim. (c) A plot of the rim radius as a function of time for the nonequilibrium double emulsion drops consisting of an inner drop of water with 16 wt. \% PEG $\left(M_{w}=4000\right)$, a middle shell of water with 5 wt. \% $\mathrm{Na}_{2} \mathrm{CO}_{3}$, and a continuous phase of silicone oil $(500 \mathrm{cSt})$. The onset of the transformation is linearly extrapolated from the experimental data due to the difficulty in identifying and measuring the very early rim radius. (d) Velocity of the rim for the same non-equilibrium drops suspended in silicone oils with different viscosity. Data and error bars are obtained from 5 randomly chosen non-equilibrium double emulsion drops. The solid lines (red) are guides to the eyes. 
and $l$ is the characteristic length of rim [see Figure S4 in the supplementary material]. Therefore, we balance the two most relevant forces in the process: the interfacial tension gradient $\left(\sim\left(\gamma_{S O}-\gamma_{P O}\right)\right)$, which drives the evolution, and the viscous drag per unit length $\left(\sim \mu_{P E G} V_{\text {rim }}\right)$, which opposes the evolution. However, when the concentrations of PEG and salts in the two aqueous solutions are low, the interfacial tension gradient should approach zero, which is difficult to be determined. Hence, to simplify, we hypothesize that the interfacial tension gradients scale with the interfacial tensions between the two aqueous phases: $\left(\gamma_{S O}-\gamma_{P O}\right) \sim \gamma_{S P}$. Therefore, the capillary velocity $\left(V_{e s t}\right)$ of the transformation can be estimated as $V_{\text {est }} \sim\left(\gamma_{S O}-\gamma_{P O}\right) / \mu_{P E G} \sim \gamma_{S P} / \mu_{P E G}$.

To confirm our hypothesis, we perform a series of experiments with ATPS made up of a PEG-rich $\left(M_{w}=8000\right)$ aqueous phase and a $\mathrm{C}_{6} \mathrm{H}_{5} \mathrm{Na}_{3} \mathrm{O}_{7} \cdot 2 \mathrm{H}_{2} \mathrm{O}$-rich aqueous phase with the interfacial tension ranging from several $\mu \mathrm{N} / \mathrm{m}$ to $2.62 \mathrm{mN} / \mathrm{m}$. As the interfacial tension of the two aqueous phases is varied by changing the concentrations of PEG and salt, the viscosity of the PEG-rich aqueous phase changes more abruptly with PEG concentration than the viscosity of the salt does with salt concentration [see Table S1 in the supplementary material]. In the subsequent analysis, we take mainly the effects from the interfacial tension of the two aqueous phases and the viscosity of the inner PEG-rich aqueous phase into account.

Based on our experiments, the expanding velocity of the rim scales with the capillary velocity, $V_{\text {rim }} \sim V_{\text {est }}$, as shown by the plot of the measured velocity of the expanding rim as a function of the estimated capillary velocity in Fig. 4. Remarkably, with the interfacial tension of the two-aqueous phases approaching several $\mu \mathrm{N} / \mathrm{m}$, the transformation speed is slowed down by almost three orders of magnitude than the systems with high interfacial tension $(\sim \mathrm{mN} / \mathrm{m})$. This log-log plot demonstrates the power-law scaling over two orders of magnitude in transformation speed, well fitted by the line with a slope of $1.02 \pm 0.10$. The slope with a value

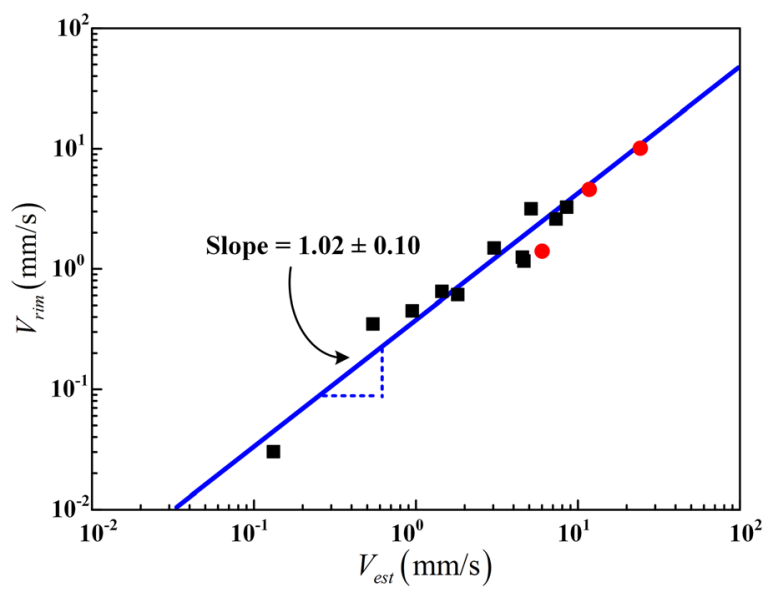

FIG. 4 . The relationship between the measured velocity $\left(V_{\text {rim }}\right)$ and the estimated capillary velocity $\left(V_{\text {est }}\right)$. The squares (black) refer to data from the ATPS consisting of PEG $\left(M_{w}=8000\right)$ and $\mathrm{C}_{6} \mathrm{H}_{5} \mathrm{Na}_{3} \mathrm{O}_{7} \cdot 2 \mathrm{H}_{2} \mathrm{O}$, while the circles (red) indicate data from the ATPS consisting of PEG $\left(M_{w}=4000\right)$ and $\mathrm{Na}_{2} \mathrm{CO}_{3}$. The solid line (blue) is a linear fit to data represented by the squares (black). The outermost phase is silicone oil (1000 cSt). The error bars are smaller than the size of filled symbols. Data and error bars are from 5 randomly chosen non-equilibrium double emulsion drops. remarkably close to unity agrees with the assumption that the interfacial tension gradients scale with the interfacial tensions between the two aqueous phases.

To further confirm the general validity of this assumption, we also perform experiments with another ATPS consisting of a PEG-rich $\left(M_{w}=4000\right)$ aqueous phase and a $\mathrm{Na}_{2} \mathrm{CO}_{3}$-rich aqueous phase. We observe that the data points, representing the relationship between the measured velocity and estimated capillary velocity, are in good agreement with the linear fitting line from the ATPS made up of PEG $\left(M_{w}=8000\right)$ and $\mathrm{C}_{6} \mathrm{H}_{5} \mathrm{Na}_{3} \mathrm{O}_{7} \cdot 2 \mathrm{H}_{2} \mathrm{O}$ solutions [Fig. 4]. The collapse of the data from another ATPS further corroborates that the transformation speed $\left(V_{\text {rim }}\right)$ of non-equilibrium w/w/o double emulsion drops towards their equilibrium can simply be estimated by the capillary velocity $\left(\gamma_{S P} / \mu_{P E G}\right)$.

In our scaling analysis for the three-phase non-equilibrium system, we greatly simplify our picture by assuming that the viscosity effects from outermost fluid can be completely ignored with respect to the dynamics of transformation. In addition, we assume the interfacial tension gradient between the two aqueous solutions and silicone is proportional to the interfacial tension of the aqueous two-phase system. These two assumptions are verified by our experimental results. However, further experiments are required to explore the transformation dynamics of non-equilibrium w/w/o double emulsion drops with a viscous shell phase.

In summary, our results show that the transformation dynamics of non-equilibrium w/w/o double emulsion drops with "complete engulfing" configuration into another equilibrium "complete engulfing" state is mainly determined by the viscosity of the innermost fluid, as well as the interfacial tension between the core and shell liquids for non-viscous middle fluids. This emphasizes that the lifetime of the nonequilibrium w/w/o double emulsions can be elongated by decreasing the interfacial tension between the core phase and shell phase or by increasing the viscosity of the innermost phase. Interestingly, the viscosity of the outermost phase has little influence on the speed of transformation. The observed dynamics of transformation will guide the choice of different emulsion phases when non-equilibrium double emulsions are used as templates for synthesizing particles with tunable morphologies.

See supplementary material for the figure illustrating the preparation of aqueous two-phase systems (ATPS), table of the physical properties of the aqueous two-phase systems used, movie showing the generation of non-equilibrium double emulsion drops, movies showing the transformation of the non-equilibrium double emulsion drops into their equilibrium morphology, figures showing the side view of transformation of the non-equilibrium double emulsions drops into equilibrium morphology, the transformation of the nonequilibrium double emulsion with paraffin liquid as the outermost phase, and the method to estimate the local Bond number.

We thank Dr. Tiantian Kong and Dr. Zhou Liu for valuable discussions. This research was supported by the Early Career Scheme (No. HKU 707712 P), the General Research Fund (Nos. HKU 719813E, 17304514, and 17306315), and 
the Collaborative Research Fund (C6004-14G) from the Research Grants Council of Hong Kong, the General Program (No. 21476189/B060201) and the Major Research plan (No. 91434202) from the National Natural Science Foundation of China, as well as the Seed Funding Programme for Basic Research (Nos. 201211159090 and 201311159105) from the University of Hong Kong.

${ }^{1}$ G. Muschiolik, Curr. Opin. Colloid Interface Sci. 12, 213 (2007).

${ }^{2}$ M. Gallarate, M. Carlotti, M. Trotta, and S. Bovo, Int. J. Pharm. 188, 233 (1999).

${ }^{3}$ C. H. Chen, A. R. Abate, D. Lee, E. M. Terentjev, and D. A. Weitz, Adv. Mater. 21, 3201 (2009).

${ }^{4}$ S. S. Datta, A. Abbaspourrad, E. Amstad, J. Fan, S. H. Kim, M. Romanowsky, H. C. Shum, B. J. Sun, A. S. Utada, M. Windbergs, S. B. Zhou, and D. A. Weitz, Adv. Mater. 26, 2205 (2014).

${ }^{5}$ A. S. Utada, E. Lorenceau, D. R. Link, P. D. Kaplan, H. A. Stone, and D. A. Weitz, Science 308, 537 (2005).

${ }^{6}$ R. K. Shah, H. C. Shum, A. C. Rowat, D. Lee, J. J. Agresti, A. S. Utada, L. Y. Chu, J. W. Kim, A. Fernandez-Nieves, C. J. Martinez, and D. A. Weitz, Mater. Today 11, 18 (2008).

${ }^{7}$ H. Chen, J. Li, J. Wan, D. A. Weitz, and H. A. Stone, Soft Matter 9, 38 (2013).

${ }^{8}$ S. Torza and S. Mason, Science 163, 813 (1969).

${ }^{9}$ J. Guzowski, P. M. Korczyk, S. Jakiela, and P. Garstecki, Soft Matter 8, 7269 (2012).
${ }^{10}$ C. X. Zhao and A. P. Middelberg, Angew. Chem., Int. Ed. 48, 7208 (2009).

${ }^{11}$ Y. Song and H. C. Shum, Langmuir 28, 12054 (2012).

${ }^{12}$ C. H. Choi, D. A. Weitz, and C. S. Lee, Adv. Mater. 25, 2536 (2013).

${ }^{13}$ M. F. Haase and J. Brujic, Angew. Chem., Int. Ed. 53, 11793 (2014).

${ }^{14}$ L. D. Zarzar, V. Sresht, E. M. Sletten, J. A. Kalow, D. Blankschtein, and T. M. Swager, Nature 518, 520 (2015).

${ }^{15}$ Q. Zhang, M. Xu, X. Liu, W. Zhao, C. Zong, Y. Yu, Q. Wang, and H. Gai, Chem. Commun. 52, 5015 (2016).

${ }^{16}$ T. Kong, Z. Liu, Y. Song, L. Wang, and H. C. Shum, Soft Matter 9, 9780 (2013).

${ }^{17}$ H. C. Shum, J. W. Kim, and D. A. Weitz, J. Am. Chem. Soc. 130, 9543 (2008).

${ }^{18}$ N. Pannacci, H. Bruus, D. Bartolo, I. Etchart, T. Lockhart, Y. Hennequin, H. Willaime, and P. Tabeling, Phys. Rev. Lett. 101, 164502 (2008).

${ }^{19}$ J. Wang, H. Jing, G. Xu, X. Wang, and Z. Duan, Appl. Phys. Lett. 107, 041602 (2015).

${ }^{20}$ H. C. Shum, J. Varnell, and D. A. Weitz, Biomicrofluidics 6, 012808 (2012).

${ }^{21}$ S. Hardt and T. Hahn, Lab Chip 12, 434 (2012).

${ }^{22}$ A. D. Diamond and J. T. Hsu, AIChE J. 36, 1017 (1990).

${ }^{23}$ E. Scholten, R. Tuinier, R. Tromp, and H. Lekkerkerker, Langmuir 18, 2234 (2002).

${ }^{24}$ B. Song and J. Springer, J. Colloid Interface Sci. 184, 64 (1996).

${ }^{25}$ B. Song and J. Springer, J. Colloid Interface Sci. 184, 77 (1996).

${ }^{26}$ A. Sauret and H. C. Shum, Appl. Phys. Lett. 100, 154106 (2012).

${ }^{27}$ B. U. Moon, S. G. Jones, D. K. Hwang, and S. S. Tsai, Lab Chip 15, 2437 (2015).

${ }^{28}$ A. R. Abate, J. Thiele, and D. A. Weitz, Lab Chip 11, 253 (2011). 